\title{
Pancreatitis aguda secundaria a hipertrigliceridemia: presentación de dos casos clínicos
}

\author{
S. J. Jiménez Forero, D. X. Roa Saavedra y M. C. Villalba \\ Unidad de Cuidado Intensivo Quirúrgico. Hospital Universitario Fundación Santa Fe de Bogotá. Colombia
}

\section{RESUMEN}

La pancreatitis aguda es un proceso inflamatorio reversible. La hipertrigliceridemia como etiología de la pancreatitis aguda varía entre un 1,3 y un $11 \%$, de acuerdo a la literatura, cuando los niveles de triglicéridos alcanzan valores por encima de $1.000 \mathrm{mg} / \mathrm{dl}$; sin embargo, la hipertrigliceridemia se observa en un 12 a un $39 \%$ de las pancreatitis agudas como factor asociado.

El objetivo del tratamiento médico es aumentar la actividad de la lipoproteinlipasa y aumentar la degradación de los quilomicrones; disminuyendo así los valores plasmáticos de triglicéridos a niveles menores de $500 \mathrm{mg} / \mathrm{dl}$, incluso a menos de $200 \mathrm{mg} / \mathrm{dl}$ si es posible con diferentes estrategias, entre ellas la insulina.

En el presente artículo, presentamos dos casos clínicos de pancreatitis severas inducidas por hipertrigliceridemia, manejadas con terapia de infusión de insulina con adecuada respuesta clínica y paraclínica con disminución significativa de los niveles de triglicéridos, 48 horas postratamiento.

Palabras clave: Pancreatitis. Hipertrigliceridemia. Hiperlipidemia.

\begin{abstract}
Acute pancreatitis is a reversible inflammatory process. Hypertriglyceridemia as a cause of acute pancreatitis reaches frequencies of $1.3-11 \%$ according to the literature when triglyceride levels reach values over $1,000 \mathrm{mg} / \mathrm{dl}$; nevertheless hypertriglyceridemia is observed in $12-39 \%$ of acute pancreatitis cases as an associated factor.

The objective of medical treatment is to increase lipoprotein-lipase activity, and to increase chylomicron breakdown thus diminishing serum triglycerides to levels smaller than 500 or even $200 \mathrm{mg} / \mathrm{dl}$ (when possible) using a variety of strategies including insulin administration.

In the present article, we report two cases of severe pancreatitis induced by hypertriglyceridemia that were managed with insulin infusion; both responded adequately, as measured by a significant reduction of triglyceride levels at 48 hours post-treatment.
\end{abstract}

Key words: Pancreatitis. Hypertriglyceridemia. Hyperlipidemia.

Jiménez Forero SJ, Roa Saavedra DX, Villalba MC. Pancreatitis aguda secundaria a hipertrigliceridemia: presentación de dos casos clínicos. Rev Esp Enferm Dig 2008; 100: 367-371.

\section{INTRODUCCIÓN}

La pancreatitis aguda es un proceso inflamatorio reversible, que puede limitarse a la afectación del órgano o de acuerdo a su severidad producir afectación multisistémica; su rango de severidad va de leve a severa y esta última tiene una tasa de mortalidad del 10-30\%,

Recibido: 03-12-07.

Aceptado: 07-12-07.

Correspondencia: Sonia Jiménez Forero. Calle 57 a no 46-34. Apto. 312. Edificio Los Conquistadores. Bogotá, Colombia. e-mail: sjjimene@urosario.edu.co dependiendo de la presencia de necrosis estéril o infectada (1).

Su etiología es muy variada, pero dentro de las principales causas se destaca la colecistitis-coledocolitiasis y el consumo de alcochol (2); la hipertrigliceridemia posee una frecuencia mucho más baja como etiología de la pancreatitis aguda con reportes entre un 1,3 y un $11 \%$, de acuerdo a la literatura, cuando los niveles de triglicéridos alcanzan valores por encima de $1.000 \mathrm{mg} / \mathrm{dl}$; sin embargo, la hipertrigliceridemia se observa en el 12 al 39\% de las pancreatitis agudas como factor asociado. Existen causas menos frecuentes como toxinas, origen metabólico, origen vascular, mecánico, infeccioso, idiopático, entre otras, con reportes de casos en la literatura. 
Para el desarrollo de pancreatitis inducida por hipertrigliceridemia, se ha reconocido que los pacientes con hiperlipidemias tipo I-IV y V de acuerdo a la clasificación de Fredrickson tienen mayor riesgo de presentarla cuando sus niveles de triglicéridos no están controlados (3).

En el presente artículo, presentamos dos casos clínicos de pancreatitis severas inducidas por hiperlipidemia, manejadas con terapia insulina con adecuada evolución y respuesta clínica y se revisan algunas de las opciones terapéuticas disponibles para su manejo.

\section{DESCRIPCIÓN DEL CASO CLÍNICO 1}

Paciente mujer de 32 años hospitalizada doce días en la unidad de cuidado intensivo, por presentar cuadro de 1 día de evolución caracterizado por epigastralgia no irradiada, la cual no cede con administración de ranitidina, valorada inicialmente en el servicio de urgencias, donde se encontró hiperamilasemia e hipertrigliceridemia $(5.080 \mathrm{mg} / \mathrm{dl})$ con ecografía de hígado y vías biliares más colangiorresonancia dentro de límites normales. Por estado de deshidratación grado III, SIRS y dolor severo, se manejó en la unidad de cuidado intensivo. Como antecedentes de importancia presentaba obesidad y dislipidemia en manejo con lovastatina $20 \mathrm{mg} /$ día y gemfibrozilo irregularmente, adicionalmente uso de productos homeopáticos (espirulina y vinagre de manzana), es una $\mathrm{G} 2 \mathrm{P} 1 \mathrm{C} 1$ y desde hace 12 años planifica con anticonceptivos orales.

$\mathrm{Al}$ examen físico de ingreso, como hallazgos relevantes se encontraron paciente somnolienta, taquicárdica, taquipneica, deshidratada; signos vitales: TA 114/70; FC: 105 por minuto; FR: 28 por min: $\mathrm{SaO}_{2}$ : $90 \%$ con $\mathrm{FIO}_{2} 28 \%$; IMC 32,5 , abdomen blando doloroso a la palpación en epigastrio sin signos de irritación peritoneal, sin otro hallazgo anormal al examen físico. Se hace diagnóstico de pancreatitis aguda severa secundaria a hipertrigliceridemia, con puntuación de 11 en APACHE inicial y 5 a las 24 horas, se realiza TAC contrastada de abdomen compatible con Baltazar E con necrosis de menos del 30\% (Fig. 1). Se manejó multidisciplinariamente con los servicios de gastroenterología y soporte nutricional, se inició infusión de insulina con descenso significativo de triglicéridos a las 48 horas (valor inicial 5.080, control a las 48 h: 369), al séptimo día se observó aumento de leucocitos, persistencia de taquicardia y PCR mayor a $16 \mathrm{mg} / \mathrm{dl}$, por lo cual se decidió iniciar cubrimiento antibiótico con meropenem. Al noveno día de hospitalización, la paciente mostraba una apariencia clínica normal sin dolor, con descenso de leucocitosis y bandemia; dada su evolución, se decide traslado a planta.

\section{DESCRIPCIÓN DE CASO CLÍNICO 2}

Paciente mujer de 38 años, ingresa a urgencias con cuadro de dolor abdominal en epigastrio y mesogastrio, de 12 horas de evolución irradiado a espalda, acompañado de emesis biliar, malestar general y disnea, sin fiebre.

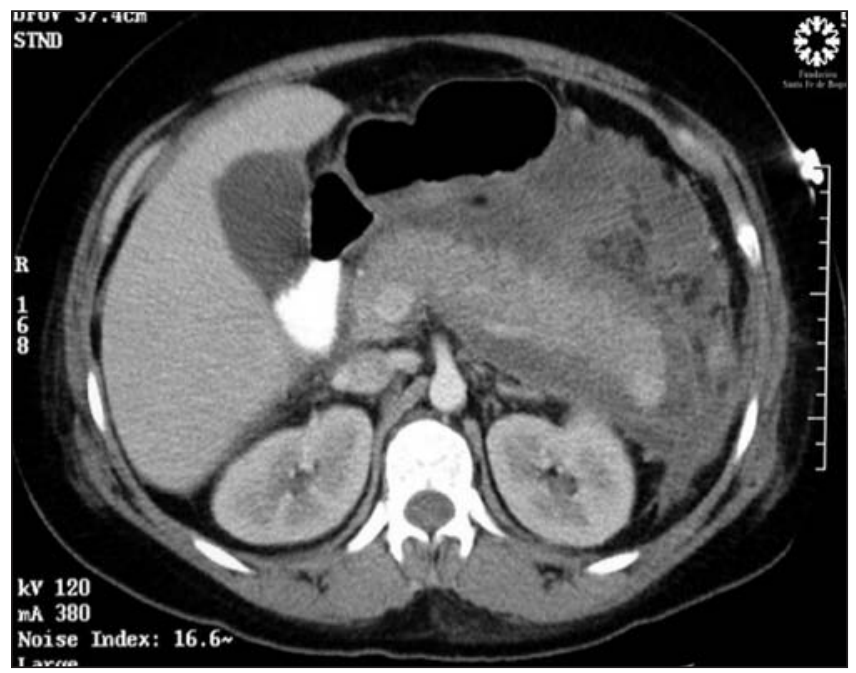

Fig. 1. Tomografía abdominal contrastada Balthazar E. Obsérvese el aumento del tamaño del páncreas, edema y líquido peripancreático.

Refiere síntomas respiratorios altos 3 semanas previas a la consulta e ingesta de comida con alto contenido graso 4 días antes. Antecedentes: diagnóstico reciente de hipotiroidismo sin tratamiento. Antecedentes quirúrgicos de: mamoplastia, lipectomía, cesárea.

$\mathrm{Al}$ ingreso al servicio de urgencias, la paciente estaba ansiosa, álgica, somnolienta, TA: 109/60; FC: 109/min; FR: 20/min; T: $37,8{ }^{\circ} \mathrm{C}$; $\mathrm{SPO}_{2}: 94 \%$ con $\mathrm{FiO}_{2}: 0,28$. Como hallazgos positivos, palidez cutánea, mucosa oral semiseca, excursión torácica limitada por dolor con hipoventilación bibasal. Abdomen distendido, blando, ruidos presentes, doloroso a la palpación profunda en epigastrio y mesogastrio, sin irritación.

Se realizan exámenes iniciales que reportan $\mathrm{Hb}: 18,9$; Hcto.: 44,3; leucocitosis de 14.000; N: 70\%; L: 22\%; Mon: 7\%; Plaq.: 252.000; sodio: 124; potasio: 3,37; cloro: 100 ; calcio: 0,86 ; magnesio: 1,9 ; creatinina: 0,58 ; BUN: 7,4; BHCG: 1,2; glicemia: 130; amilasa: 537. Pruebas de coagulación normales. Suero lipémico.

Ecografía abdominal: aumento de ecogenicidad periportal, páncreas aumentado de tamaño y aumento difuso de su ecogenicidad, presencia de líquido peripancreático, no litiasis. Se hace diagnóstico de pancreatitis aguda, se inician estudios para aclarar etiología, manejo del dolor e hidratación sin mejoría; persiste con tendencia a hipotensión, somnolencia, disminución de volúmenes urinarios y se decide traslado a unidad de cuidado intensivo (UCI). Requiere soporte inotrópico con dopamina y norepinefrina, con titulación de morfina para control de dolor.

Reporte de laboratorios complementarios: PCR: 6,5; colesterol total: $1.029 \mathrm{mg} / \mathrm{dl}$; triglicéridos: $7.508 \mathrm{mg} / \mathrm{dl}$; lipasa: $6.660 \mathrm{u} / \mathrm{l}$. Gases arteriales: $\mathrm{pH}: 7,33 ; \mathrm{PO}_{2}: 77 \%$; $\mathrm{PCO}_{2}: 26 ; \mathrm{HCO}_{3}: 13 ; \mathrm{DB}:-10$. Se considera como causa de la pancreatitis la hipertrigliceridemia y se adiciona al manejo infusión de insulina a 2 u/hora. El APACHE de ingreso 19 para una pancreatitis severa se deteriora a las 24 horas con un APACHE de 38. 
Se realiza control de triglicéridos a las doce horas de inicio de infusión de insulina con descenso marcado de los niveles a $2.224 \mathrm{mg} / \mathrm{dl}$ y colesterol total a 674 , normalizándose sus valores al día 5 de tratamiento (Fig. 2). La paciente presenta deterioro de mecánica respiratoria, disminución de $\mathrm{PaFiO}_{2}$, requiriendo intubación endotraqueal e inicio de ventilación mecánica. A las 48 h de ingreso desarrolla hipertensión intraabdominal con valores de $32 \mathrm{mmHg}$, insuficiencia renal aguda y criterios para SDRA, requiere laparotomía de urgencia, observándose páncreas de aspecto edematoso, áreas de necrosis, se realiza lavado de cavidad peritoneal sin complicaciones.

Se inicia cubrimiento antibiótico con meropenem a $1 \mathrm{~g}$ endovenoso $\mathrm{c} / 8$ horas y se decide inicio de terapia inmunomoduladora con inmunoglobulina IGM humana (5\%) Pentaglobin $^{\circledast}, 16,8 \mathrm{Ml} / 1$ hora vía i.v., teniendo en cuenta el compromiso multisistémico que presenta la paciente.

Requirió lavado de cavidad peritoneal en seis oportunidades y se colocó sistema de vacío continuo abdominal (VAC). Requirió soporte nutricional enteral y trasfusión de hemoderivados.

A partir del día ocho de ingreso inicia recuperación paulatina, estabilización hemodinámica, mejoría y resolución de fallo orgánico múltiple. El día de 10 de estancia es extubada exitosamente. Se realiza nuevo lavado el día 15 de su hospitalización, tomando muestras de líquido ascítico, en

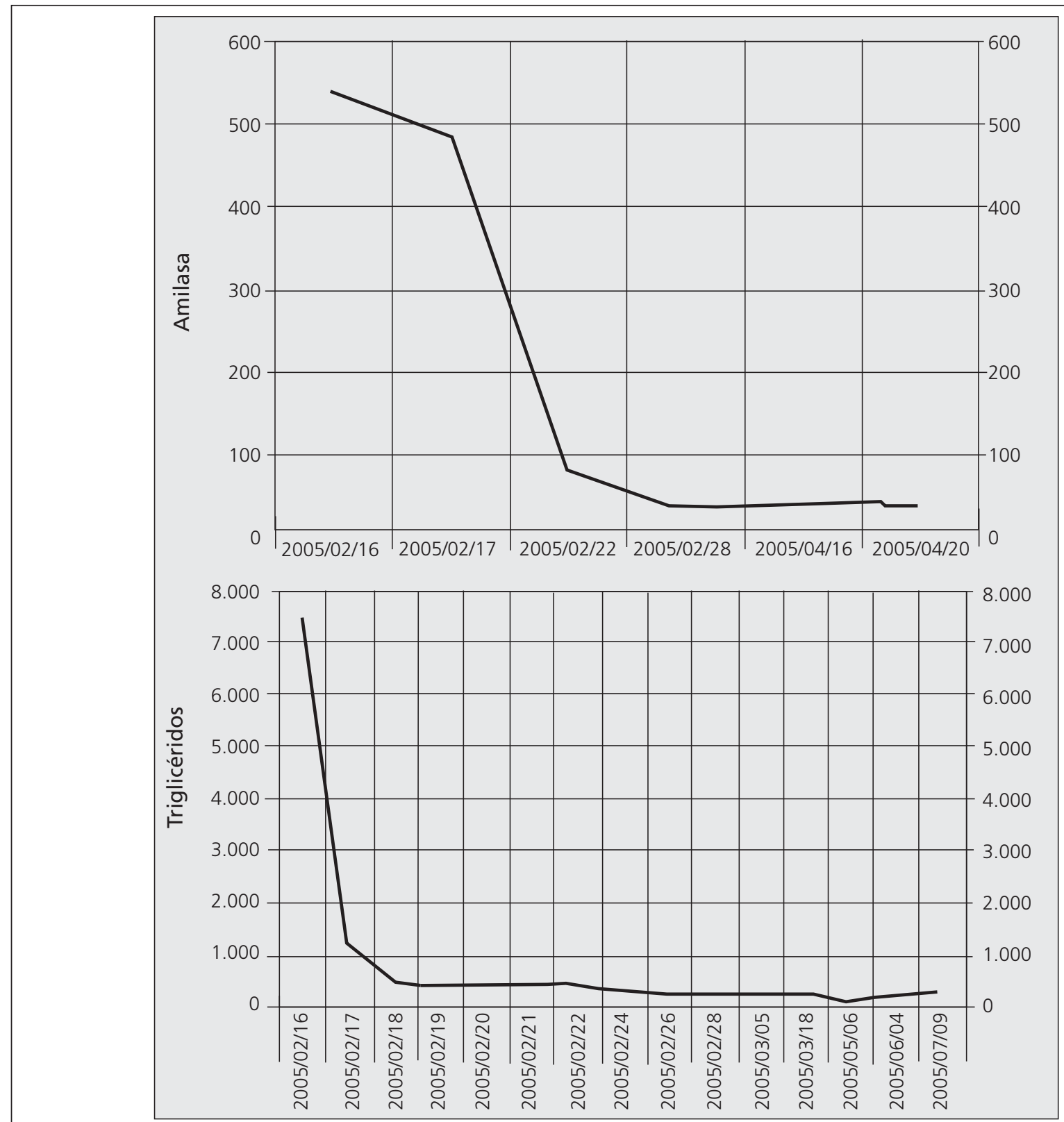

Fig. 2. Comportamiento amilasa y triglicéridos caso 2. 
el cual se obtiene crecimiento de S. epidermidis oxacilinoresistente, por lo que se inicia manejo con vancomicina. Evolución a la mejoría, tras 16 días de estancia en UCI se ordena traslado a piso, donde continúa manejo antibiótico hasta su egreso tras 32 días de estancia hospitalaria, los valores de triglicéridos están en $220 \mathrm{mg} / \mathrm{dl}$; colesterol total: 159 mg/dl; HDL 28 mg/dl; y LDL 87 mg/dl.

\section{DISCUSIÓN}

La pancreatitis aguda es una de las complicaciones más temidas de la hipertrigliceridemia severa, pudiendo llegar a ser fatal. Como se observó en los casos descritos, se trata de una patología cuya presentación y complicaciones no difieren respecto a la asociada a litiasis o alcohol, su diagnóstico se realiza generalmente tras estudios negativos para las etiologías más comunes, llevando al estudio de lípidos en sangre.

La hiperlipidemia es un hallazgo frecuente en los pacientes con pancreatitis aguda de etiología biliar o alcohólica y generalmente se considera un epifenómeno, en estos casos los niveles son bajos entre 2-10 $\mathrm{mmol} / \mathrm{lt}$ (valor normal unidades SI 0,45-1,69 mmol/litro, factor de conversión x 0,01129); cuando el origen es la hipertrigliceridemia los niveles generalmente exceden los $20 \mathrm{mmol} / \mathrm{lt}$; sin embargo el riesgo de desarrollo de la enfermedad se ha reconocido que inicia con niveles mayores a 11,3 mmol/lt (4). En pacientes que desarrollan la patología con valores más bajos se debe interrogar un defecto primario genético o del metabolismo, así como etiologías secundarias como el hipotiroidismo. Con niveles de triglicéridos menores a $5,65 \mathrm{mmol} /$ litro no se considera pancreatitis inducida por hiperlipemia, sino epifenómeno (5).

Esta patología es más frecuente en la paciente embarazada, principalmente en el segundo al tercer trimestre, donde representa aproximadamente el $56 \%$ de todas las pancreatitis presentadas en el embarazo (6).

El diagnóstico al ingreso puede ser confuso o retrasarse, al presentarse con mayor frecuencia el cuadro clínico inicial con niveles de amilasa normales o discretamente elevados, situación explicada por una inhibición de la actividad enzimática inducida por la hipertrigliceridemia, así como posibles inhibidores de la amilasa circulantes, lo que retrasaría el inicio del tratamiento $(7,8)$.

Tan importante como diagnosticar la etiología de la pancreatitis es determinar la etiología de la hipertrigliceridemia, esta puede ser primaria o, con menor frecuencia, secundaria a obesidad, alcoholismo, hipotiroidismo o exacerbación de una hipertrigliceridemia preexistente con el embarazo, inducida por fármacos como tamoxifeno, entre otros. El diagnóstico del origen de la hipertrigliceridemia complementa el manejo.

\section{Fisiopatología}

Se han descrito varios mecanismos causales de la aparición de pancreatitis en los pacientes con hiperlipidemia.
Un primer mecanismo está explicado por el daño directo de los ácidos grasos al tejido pancreático; generalmente estos no son tóxicos unidos a la albúmina, pero en la hipertrigliceridemia se supera la capacidad de la albúmina para su transporte, aumentando su toxicidad y desencadenando la respuesta enzimática en el páncreas tras su degradación por la lipasa endotelial y la lisolecitina en el tejido acinar (9).

Un segundo mecanismo se explica por los quilomicrones, los cuales pueden obstruir la circulación distal pancreática causando isquemia (descrito en hiperlipidemia tipo I donde se altera la reología sanguínea), esto causa una alteración de la arquitectura acinar, se expone el tejido pancreático a los triglicéridos activando la lipasa pancreática, desencadenando la cadena inflamatoria y un ciclo de mayor activación de todo el grupo de enzimas pancreáticas. Igualmente los ácidos grasos libres en altas concentraciones crean un medio ácido, este cambio en el $\mathrm{pH}$ puede desencadenar la activación del tripsinógeno y el inicio de la enfermedad.

Un tercer mecanismo se asocia a una disminución genética de la lipoproteinlipasa (LPL), generalmente de carácter autosómico recesivo. Esta enzima se sintetiza en el tejido muscular y en los adipocitos. Tras su liberación es transportada al endotelio capilar, allí actúa sobre los quilomicrones ricos en triglicéridos, hidrolizándolos a quilomicrones remanentes, e hidroliza las lipoproteínas ricas en triglicéridos a ácidos grasos y glicerol, productos que son transportados al hígado, músculo esquelético, miocardio y adipocitos para su utilización. Su déficit produce una alteración de la degradación plasmática de los quilomicrones en el suero de estos pacientes respecto a otros controles (10).

Existen otras alteraciones, como el caso del hipotiroidismo, donde se altera el metabolismo de las lipoproteínas así como la actividad del receptor para LDL, lo que predispone a elevación de sus niveles en suero. En este caso se aumenta el riesgo de pancreatitis cuando hay una descompensación aguda de esta patología o un aumento de los requerimientos basales de hormona tiroidea en el paciente.

\section{Tratamiento}

El tratamiento de la hipertrigliceridemia está indicado tanto en la pancreatitis inducida por esta etiología como en los casos de pancreatitis de otros orígenes, como el biliar, donde como epifenómeno, puede exacerbar la severidad del cuadro clínico. No está descrito un tratamiento estándar para el manejo de la enfermedad, es poca la literatura disponible, la mayoría son reportes de casos, dado que no es una patología frecuente, las guías disponibles son escasas (11), faltan estudios adicionales y estandarizar las recomendaciones disponibles.

Teniendo en cuenta las limitaciones descritas, el tratamiento es conservador, buscando la disminución de los niveles de triglicéridos y previniendo el desarrollo de compli- 
caciones, como disfunción multiorgánica o sepsis (12). El objetivo del tratamiento médico es aumentar la actividad de la lipoproteinlipasa y aumentar la degradación de los quilomicrones (13); disminuyendo así los valores plasmáticos de triglicéridos a niveles menores a 500, incluso a menores de $200 \mathrm{mg} / \mathrm{dl}$ si es posible con diferentes estrategias.

El ayuno tiene un papel primordial en este tipo de pancreatitis, debido a que este favorece el rápido metabolismo de los quilomicrones y de los triglicéridos, cuya fuente principal es la ingesta de grasas. Con el tratamiento, los niveles descienden generalmente los primeros 3 días, como lo observado en los casos clínicos, algunos reportes hablan de disminuciones rápidas en las primeras 24 horas, pero se han descrito niveles elevados hasta por 15 días de acuerdo al trastorno de base y su severidad.

Dentro de las opciones terapéuticas descritas están la insulina, heparina, plasmaféresis y apoproteína C2 purificada. La insulina y la heparina constituyen las opciones terapéuticas con mayor descripción, por su fácil disponibilidad (Tabla I); estas inducen la actividad de la lipoproteinlipasa plasmática ligada al endotelio, adicionalmente la heparina moviliza esta enzima desde el endotelio al plasma y la insulina favorece la degradación de los quilomicrones. La plasmaféresis se ha descrito en las formas agudas, así como de forma profiláctica en los casos crónicos, donde el manejo nutricional y farmacológico no ha sido óptimo y persiste el riesgo para pancreatitis. La plasmaféresis en los casos agudos no sólo disminuye los niveles plasmáticos de triglicéridos, sino que realiza un barrido de varias citoquinas proinflamatorias plasmáticas y proteasas, así como disminuye la viscosidad plasmática aumentando la perfusión del tejido.

\section{Tabla I. Medicamentos disponibles para manejo y dosis propuestas}

\begin{tabular}{|c|c|}
\hline Medicamento & Dosis \\
\hline Heparina & $\begin{array}{l}10.000 \text { unidades para } 24 \mathrm{~h} \\
5.000 \text { unidades s.c. cada } 12 \text { a } 24 \mathrm{~h}\end{array}$ \\
\hline Insulina & $\begin{array}{l}\text { Mantener niveles de glicemia menores a } 200 \mathrm{mg} / \mathrm{dl} \\
\text { Infusión continua de insulina cristalina de acuerdo a metas de glu- } \\
\text { cometría } \\
\text { Inyección única diaria de } 0,1 \text { uk/día }\end{array}$ \\
\hline Antioxidantes & $\begin{array}{l}\text { Selenio, betacarotenos, vitamina C y tocoferol ofrecen protección } \\
\text { a las células acinares tras el daño iniciado }\end{array}$ \\
\hline
\end{tabular}

Aún no se conoce la dosis óptima de estos tratamientos y cuál representa mayor beneficio de acuerdo a la severidad del cuadro clínico. Incluso hay casos descritos de manejo conservador sin medidas específicas, logrando disminución de los niveles de triglicéridos y otros como el uso de terapias de la medicina tradicional china por los doctores EnQiang y colaboradores, con resultados satisfactorios, proponiendo en sus guías la penta-association therapy.

Siempre es necesario tener en cuenta otras medidas como inicio temprano de derivados del ácido fíbrico, tratar la causa secundaria si es el desencadenante, prevenir la recurrencia con controles periódicos y tratamiento multimodal, manejar los factores de riesgo asociados como diabetes, hipotiroidismo, abuso de alcohol, entre otras medidas.

\section{CONCLUSIONES}

La pancreatitis aguda inducida por hipertrigliceridemia es una entidad recientemente descrita, que requiere un rápido diagnóstico para iniciar un enfoque terapéutico. No se conoce en la actualidad evidencia suficiente para indicar una guía terapéutica, el manejo ideal no está determinado. Surgen entonces preguntas, como cuáles serán las indicaciones de plasmaféresis en los casos agudos; de acuerdo a los adelantos respecto al estricto control de la glicemia, la insulina beneficia a estos pacientes por una verdadera inducción de la lipoproteinlipasa o sus beneficios estarán más relacionados con el control de los niveles de la glicemia.

Consideramos importante medir en todos los pacientes con pancreatitis aguda los niveles de triglicéridos, dado que la hiperlipidemia también se presenta como epifenómeno en esta entidad, que puede empeorar el manejo de la patología de base.

\section{BIBLIOGRAFÍA}

1. Werner J, Feuerbach S, Uhl W, Büchler MW. Management of acute pancreatitis: From surgery to interventional intensive care. Gut 2005; 54 (3): 426-36.

2. Caroll J, et al. Acute pancreatitis: Diagnosis, prognosis and treatment. Am Fam Physician 2007; 75 (10): 1513-20.

3. Yadav D, Pitchumoni CS. Issues in hyperlipidemic pancreatitis. J Clin Gastroenterol 2003; 26: 54-62.

4. Yeh JH, Chen JH, Chiu HC. Plasmapheresis for hyperlipidemic pancreatitis. J Clin Apher 2003; 18: 181-5.

5. Okerberg K, Lee M. Spuriously normal amylase levels in a patient with acute pancreatitis secondary to hypertriglyceridemia. J Am Board Fam Pract 1999; 12: 68-70.

6. Chang CC, Hsieh YY, Tsai HD, Yang TC, Yeh LS, Hsu TY. Acute pancreatitis in pregnancy. Zhonghua Yixue Zazhi (Taipei) 1998; 61: 85-92.

7. Fallat RW, Vester JW, Glueck CJ. Suppression of amylase activity by hypertrigliceridemia. JAMA 1973; 225: 1331-4.

8. Okura Y, Hayashi K, Shingu T, Kajiyama G, Nakashima Y, Saku K. Diagnostic evaluation of acute pancreatitis in two patients with hypertriglyceridemia. World J Gastroenterol 2004; 10 (24): 3691-5.

9. Zoltán Berger F, Rodrigo Quera P, Jaime Poniachik T, Danny Oksenberg R, Julia Guerrero P. Heparina e insulina en el tratamiento de la pancreatitis aguda por hipertrigliceridemia. Experiencia en 5 casos. Rev Méd Chile 2001; 129 (12): 1373-8.

10. Gan SI, Edwards AL, Symonds CJ, Beck PL. Hypertriglyceridemiainduced pancreatitis: A case-based review. World J Gastroenterol 2006; 12 (44): 7197-202.

11. En-Qiang M, Yao-Qing T, Sheng-Dao Z. Formalized therapeutic guideline for hyperlipidemic severe acute pancreatitis. World J Gastroenterol 2003; 9: 2622-6.

12. Kyriakidis AV, Raitsiou B, Sakagianni A, Harisopoulou V, Pyrgioti M, Panagopoulou A, et al. Management of acute severe hyperlipidemic pancreatitis. Digestion 2006; 73: 259-64.

13. Alagözlü H, Cindoruk M, Karakan T, Ünal S. Heparin and insulin in the treatment of hypertriglyceridemia-induced severe acute pancreatitis. Dig Dis Sci 2006; 51: 931-3. 